\title{
The promise of surgery, its scope and limitations in leprosy
}

\author{
DINKER D. PALANDE \\ 25 and 27 Advocate Chinnathambi Street, Kurichikuppam, \\ Pondicherry-12, India 605012
}

Accepted for publication 22 April 1998

\section{Introduction}

Leprosy is a systemic disease mainly manifested in skin and nerves. The nerves affected are the cutaneous and trunk nerves, especially those located superficially. Early treatment of the disease prevents deformity or disability. Otherwise, the continuing disease process and immune reaction, either by direct involvement of tissues or secondarily through destruction of some trunk nerves, results in deformities and disabilities. All of these complications are amenable to surgical treatment, the results being excellent when the surgery is performed early.

To a deformed patient, appearance means the difference between being an outcast and a normal life in the community. To leprosy control programmes, surgical treatment means the possibility of offering a comprehensive treatment to those cases needing more than chemotherapy. For this reason, surgery is an important part of leprosy treatment and should be available to all control programmes.

Health Education (HE) and Prevention of Disabilities (POD) are also essential activities to all leprosy control programmes. Although these activities show a more adequate cost/ benefit ratio, surgery should also be regarded as a preventive measure. As examples, surgical correction of drop foot prevents the onset of distorted foot and ulcers in the lateral border which usually leads to a total destruction of the foot. Appropriate surgical correction of clawed hands prevents rigidity of small joints in the affected hand and also improves the grasp, reducing the possibility of wounds in finger tips which, otherwise, would lead to a grossly deformed and useless hand.

- Leprosy may cause motor paralysis and loss of sensation, leading to disabilities and deformities. Surgery can correct most of the disabilities due to motor paralysis.

- Surgery should be available as part of the whole treatment of leprosy patients.

- Doctors should be aware of the possibilities of surgery in leprosy in order to refer cases in need.

\section{The promise of surgery}

Surgical treatment of deformities in leprosy ensures optical restoration of function and 
appearance. Proper and adequate surgery done early offers the best results. Surgery is indicated when non-surgical treatment proves inadequate. It is important to note that reconstructive surgery aims to restore some function-that is lost due to paralysis of some muscles-to the affected hand or foot; but no sensory recovery can be expected from such procedures. This must be clearly explained by doctors and understood by patients before any rehabilitation programme is settled, in order to prevent overexpectation in terms of results.

Health Education and Prevention of Disabilities measures must be permanent for patients undergoing reconstructive surgery in leprosy, since reconstructive procedures in leprosy do not aim to restore normal sensation. It aims only to improve the motor function to the paralysed group of muscles and/or cosmetic appearance.

\section{The scope and limitations of surgery in leprosy}

Table 1 shows the cause, consequence and the available surgical treatment of these conditions in leprosy, while Figure 1 shows the deformity.

All operations, except tendon transfers, are common procedures and can be done by any surgeon familiar with them. They all give excellent results. Tendon transfer surgery is specialized, not normally available, and its success needs additional input of pre- and postoperative training through physical therapy and a well-motivated patient. The knowledge and methods to prevent ulcer recurrence also need to be specially provided.

Treatment of plantar ulcers is simple; prevention of ulcers and of their recurrence requires special knowledge and skills.

Healing a plantar ulcer is no different from healing any other wound or ulcer. The

Table 1. Cause, consequence and available surgical treatment in leprosy

\begin{tabular}{|c|c|c|}
\hline Cause & Consequence & Surgical treatment \\
\hline Trunk nerve involvement with compression & Increasing loss of nerve function & Nerve decompression \\
\hline $\begin{array}{l}\text { Loss of sensation secondary to nerve } \\
\text { involvement (prevention of ulcers and of } \\
\text { their recurrence is a specialized subject) }\end{array}$ & $\begin{array}{l}\text { Ulcers on soles of feet and palms of } \\
\text { hand; tendon and bone infection } \\
\text { Secondary deformity of foot and } \\
\text { fingers }\end{array}$ & $\begin{array}{l}\text { Debridgement, skin } \\
\text { grafting, release of } \\
\text { focus of infection } \\
\text { Arthrodeses etc. }\end{array}$ \\
\hline \multicolumn{3}{|l|}{ Loss of motor power by nerve involvement } \\
\hline Facial nerve & Lagophthalmos & Temporalis transfer \\
\hline Ulnar, median & 'Claw' deformity of fingers and thumb & Tendon transfers \\
\hline Radial and triple nerve paralysis & $\begin{array}{l}\text { Wrist drop } \\
\text { Completely deformed and dysfunctional } \\
\text { hand }\end{array}$ & Arthrodeses \\
\hline Posterior tibial nerve & Clawing of toes of grades $1,2 \& 3$ & $\begin{array}{l}\text { Tendon transfer } \\
\text { Arthrodeses }\end{array}$ \\
\hline Lateral poplitcal nerve & Complete or partial 'foot-drop' & Tendon transfer \\
\hline Direct tissue involvement & $\begin{array}{l}\text { Ear lobe deformities } \\
\text { Nasal deformities } \\
\text { Gynaecomastia }\end{array}$ & $\begin{array}{l}\text { Plastic surgery } \\
\text { (reconstructive) }\end{array}$ \\
\hline
\end{tabular}




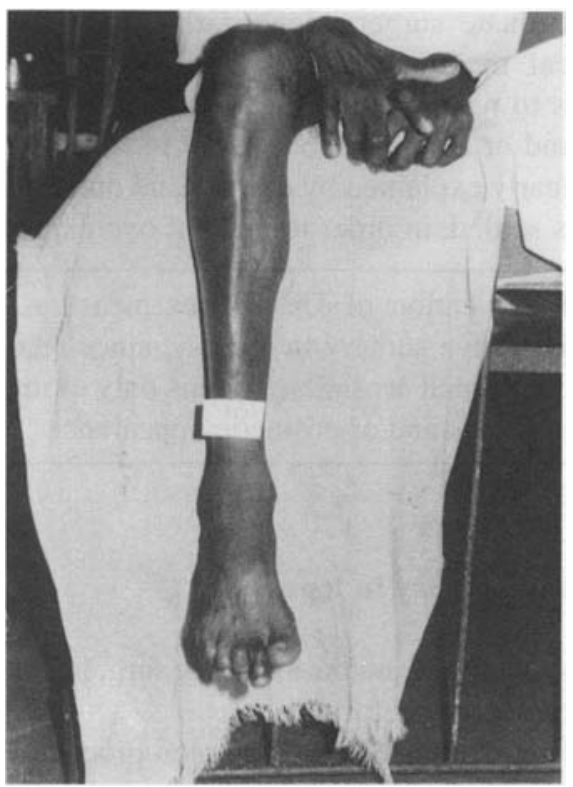

Figure 1. Deformities of hands (ulnar and median paralysis) and foot (lateral poplitcal and posterior tibial nerve paralysis).

principle of treatment is to make a complicated ulcer simple by removing all sources of infection. This can be done by any trained doctor or surgeon. Prevention of ulcer recurrence, a part of any prevention of disability programme, requires the following for implementation: (1) knowledge of why ulcers occur on a sole which has loss of sensation and even more so when there is also paralysis of intrinsic muscles; (2) knowledge and skill of a podiatrist and a cobbler to ensure good distribution of weight bearing and protection of any vulnerable part. The methods of prevention of ulcer recurrence are:

1. Health education by transfer of knowledge as to why ulceration occurs and how to prevent ulcers by self-care measures.

2. Provision of simple devices such as arch support and heel and metatarsal pads to protect vulnerable areas of the plantar surface of the foot.

3. Provision of specialized footwear and foot care. The special footwear distributes pressure, protects scars and prevents injury. Thus collaboration with a health educator, a foot specialist, podiatrist and shoemaker is essential.

\section{Tendon transfers}

\section{RATIONALE FOR TENDON TRANSFERS}

Any movement pattern, e.g. writing, is learned, and involves well orchestrated contraction of different muscles and dynamic play of balance of forces across joints. When some muscles are paralysed, there is an imbalance in these muscular forces. This is corrected by relocation of tendon(s) of normal muscles, called tendon transfer. The function of these transferred 
muscles has to be learned by the brain, in order to produce the required movement pattern. This is achieved by a planned training programme using principles of physical and occupational therapy and teaching techniques, not only before and soon after surgery, but also during the periodic follow-up visits for at least 1 year.

\section{SURGICAL PROCEDURE}

Tendon transfer surgery, as treatment of motor paralysis, though also performed for nonleprosy patients, is rather uncommon outside the field of leprosy and hence not normally available in an ordinary hospital. A specialized, well-trained team is needed, as in transplant surgery, not only for the operation itself but also for the continued postoperative monitoring, to ensure a sustained long-term result. Its success requires not only a special surgical set-up and technique, but also a learning programme for the patient, with input from physical/ occupational therapy and teaching techniques. For this reason, training as a general surgeon is not sufficient, and any surgeon who performs this kind of surgery only occasionally is unlikely to achieve consistently good results. The results of tendon transfer surgery on the hand is shown in Figure 2.

Pre-requisites for surgery for paralytic conditions

1. Well-motivated patient, trained surgeon and physiotherapist.

2. Patient under treatment for leprosy.

3. Normal mobility of fingers or thumb or foot.

4. BI should be 2 or less.

5. Well stabilized disease and reaction free.

6. No focus of infection.
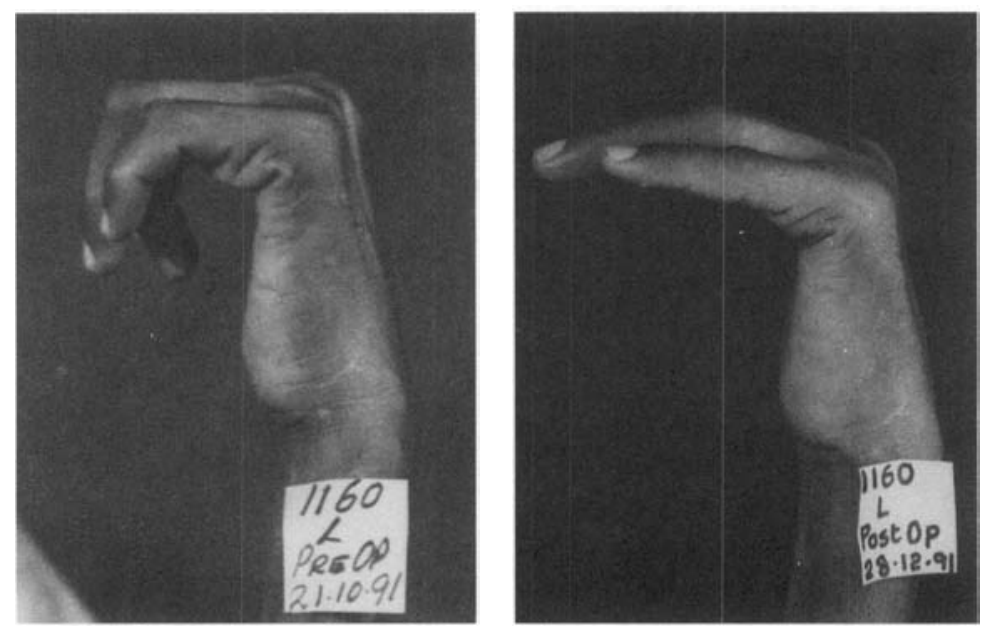

Figure 2. Results of tendon transfer, repair of combined ulnar/medium paralysis. Palande's procedure, five-tailed extensor carpi radialis longus transfer to the intrinsic muscles. (a) Pre-operative; (b) postoperative. 
PRE- AND POSTOPERATIVE TRAINING

Special training is exemplified by teaching isolated contraction of the muscle-tendon unit to be transferred, before and after surgery. For example, in the transfer of a wrist extensor to the fingers for correction of clawed fingers, the following training steps may be employed:

1. Isolation of the extensor carpus radialis longus (ECRL) before surgery by teaching the patient to contract, see and become aware of the tendon and its action of wrist extension and radial deviation.

2. Postoperatively, at first, the patient is asked to contract the ECRL (as learned preoperatively) while blindfolded. Only the observing therapist is aware of what is happening when the ECRL contracts.

3. When the action of the tendon is well established, the patient performs the same exercise of contracting the transfer, but with eyes open. The patient is always surprised to find that now, on contraction of the ECRL, instead of wrist extension, the fingers are flexing at the metacarpal joints! Slowly, this new action becomes a part of normal activity.

4. This integration is hastened by training the patient to perform purposeful movements such as writing and picking of objects and, in India, relearning the correct way to eat with the hand.

5. In follow-up visits, all of the above actions are repeatedly reinforced. The patient is first asked to show how the transfer is working, and then the training is repeated each time. The timetable for these visits is as follows:

i. Pre-operative physiotherapy: 1-3 weeks, depending on the condition of the hand.

ii. Operation. Postoperative immobilization for 3-4 weeks.

iii. Postoperative treatment: 4-5 weeks. No heavy work for another 3 months. This is to protect the transfer.

iv. Follow-up visits: 15 days, then once monthly for 6 months, every 2 months for the next 6 months, then every 3 months for 5 years.

The aim of tendon transfer operations is optimum restoration of functional ability, and of appearance. Normal power and function can neither be expected nor achieved. It is essential to maintain normal mobility of the fingers without injuries, scars and contractures until surgery can be done, and similar maintenance and care after surgery in order to obtain and sustain good results. The success of surgery in leprosy requires knowledge and motivation on the part of both the patient and the team managing and treating them. All those working in the field of leprosy need to know the limitations and scope of leprosy surgery. 